\title{
Effect of Chaihu-Shugan-San on the mRNA expression of the 5-HT1A receptor and cellular proliferation in the hippocampus of epileptic rats with depression
}

\author{
PING YANG ${ }^{1 *}$, LIANG LI ${ }^{2,3^{*}}$, XUE-JUN LIU ${ }^{1}$, XIONG CAI $^{2}$, MEI-ZHEN SUN ${ }^{4}$, JUN-FENG HE $^{2}$, \\ GUANG ZENG $^{2}$ and HUI-YONG HUANG ${ }^{2}$
}

\author{
${ }^{1}$ Department of Psychiatrics, Hunan Brain Hospital, Changsha, Hunan 410007; ${ }^{2}$ Provincial Key Laboratory of \\ TCM Diagnostics and ${ }^{3}$ Department of Anatomy, Hunan University of Chinese Medicine, Changsha, Hunan 410208; \\ ${ }^{4}$ Department of Neurology, The First Hospital of Shanxi Medical University,
} Taiyuan, Shanxi 030001, P.R. China

Received October 4, 2014; Accepted September 29, 2015

DOI: $10.3892 / \mathrm{etm} .2015 .2867$

\begin{abstract}
Chaihu-Shugan-San (CHSGS) is a herbal preparation that has been shown to effectively relieve neurologic impairment and reduce depression. However, the efficacy of CHSGS in the treatment of patients with epilepsy with depression is unknown. Therefore, in the present study, adult rats were exposed to chronic mild stress following the establishment of chronic pilocarpine-induced epilepsy. Subsequently, a sucrose consumption test and open-field test (OFT) were performed to assess depression-like behavior. Rats were randomly divided into four groups: Control, model, fluoxetine $(1.8 \mathrm{~g} / \mathrm{kg})$ and CHSGS $(2.7 \mathrm{~g} / \mathrm{kg})$ groups. The control and model groups received normal saline. The mRNA expression levels of the 5-hydroxytryptamine 1A (5-HT1A) receptor and the number of 5-bromo-2'-deoxyuridine (BrdU)-labeled cells in the hippocampal dentate gyrus were detected using reverse transcription-quantitative polymerase chain reaction and immunohistochemical analysis, respectively. Treatment administration was conducted by once daily intragastric perfusion for 28 days. The mRNA expression levels of the 5-HT1A receptor, the number of BrdU-labeled cells in the hippocampal dentate gyrus, the consumption of sucrose, and frequency of
\end{abstract}

Correspondence to: Professor Hui-Yong Huang, Provincial Key Laboratory of TCM Diagnostics, Hunan University of Chinese Medicine, 300 Xueshi Road, Hanpu Science and Education Park, Changsha, Hunan 410208, P.R. China

E-mail: huanghy68@126.com

${ }^{*}$ Contributed equally

Abbreviations: CHSGS, Chaihu-Shugan-San; CMS, chronic mild stress; OFT, open-field test; 5-HT1A, 5-hydroxytryptamine 1A; 8-OH-DPAT, 8-hydroxy-dipropylaminotetralin; SD, Sprague-Dawley

Key words: Chaihu-Shugan-San, 5-hydroxytryptamine 1A receptor, cell proliferation, epilepsy with depression, chronic mild stress vertical and horizontal movement scores in the OFT were enhanced in the fluoxetine and CHSGS groups compared with the model group $(\mathrm{P}<0.05)$. However, no statistically significant difference was detected between the fluoxetine and CHSGS groups. These data suggest that CHSGS is able to increase the expression of 5-HT1A receptor mRNA and cellular proliferation in the hippocampal dentate gyrus in epileptic rats with depression, and thus effectively improve certain symptoms of depression.

\section{Introduction}

The co-occurrence of epilepsy and depression is commonly observed in neurological psychiatry (1). Studies have revealed that the co-occurrence of epilepsy with depression is associated with the activation of 5-hydroxytryptamine $1 \mathrm{~A}$ (5-HT1A) receptors $(2,3)$. A previous study indicated that the 5-HT1A receptor agonist 8-hydroxy-dipropylaminotetralin (8-OH-DPAT) exerts anti-epileptic and anti-depressive effects in epileptic rats with depression, which involve the enhancement of the growth of hippocampal neurons (4). However, certain current treatments possess shortcomings, such as the antidepressant bupropion which may cause generalized seizures and central nervous system depression if administered in excess (5). Chinese medicinal herbs, such as Bupleurum chinense root and Pericarpium Citri Reticulatae appear to exhibit limited adverse reactions and therapeutic efficacy (6).

The present study employed chronic epileptic rats with depression induced by a combination of pilocarpine and chronic mild stress (CMS) to investigate the efficacy of Chaihu-Shugan-San (CHSGS). Detection methods included behavioral analysis, immunohistochemistry and reverse transcription-quantitative polymerase chain reaction (RT-qPCR). The expression levels of 5-HT1A receptor mRNA and numbers of 5-bromo-2'-deoxyuridine (BrdU)-labeled cells in the hippocampus were detected to clarify whether CHSGS improves cellular proliferation in the hippocampus by promoting the expression of 5-HT1A receptor mRNA. The results of this 
study may support the use of CHSGS as a novel treatment for epilepsy with depression.

\section{Materials and methods}

Animals and experimental protocol. The present study was a randomized, controlled animal study. The study was performed at the Anatomical Research Laboratory of Hunan University of Chinese Medicine (Changsha, China). A total of 60 male Sprague-Dawley (SD) rats (weight, 180-220 g; age, 8-9 weeks) were provided by the Laboratory Animal Center of Hunan University of Chinese Medicine [License no. SYXK(Xiang)2009-0001; Changsha, China]. Rats were separately housed in plastic cages at $22^{\circ} \mathrm{C}$ and $60 \%$ humidity under a constant light-dark cycle and were allowed free access to food and water. The animals were acclimatized to the environment for 2 weeks, while being trained to consume $1 \%(\mathrm{w} / \mathrm{v})$ sucrose solution for taste adaptation. Based on the baseline scores of sucrose consumption test and the open-field test (OFT) during the adaptation period, 48 rats with similar behavior were randomly divided into four groups: Control, model, fluoxetine and CHSGS groups ( $n=12$ per group). All procedures were performed in accordance with the Guidance Suggestions for the Care and Use of Laboratory Animals, formulated by the Ministry of Science and Technology of China (7).

Reagents. The CHSGS preparation contained the following: $6 \mathrm{~g}$ Bupleurum chinense root, $6 \mathrm{~g}$ Pericarpium Citri Reticulatae, 4.5 g Rhizoma Chuanxiong, 4.5 g Rhizoma Cyperi, 4.5 g Fructus Aurantii, 4.5 g Paeonia and $1.5 \mathrm{~g}$ Glycyrrhizae uralensis root. All CHSGS herbal components were obtained from the First Hospital of Hunan University of Chinese Medicine. The herbal components were identified by an expert to fulfill the quality requirements of the Pharmacopoeia of the People's Republic of China (8). CHSGS and its components were individually decocted in boiling water for $45 \mathrm{~min}$, concentrated and vacuum-dried to form a paste, and were subsequently combined into a paste containing $8 \mathrm{~g}$ crude extracts per gram. This CHSGS paste was diluted with distilled water to a concentration of $1.0 \mathrm{~g} / \mathrm{ml}$ for application. The CHSGS administration dose was $2.7 \mathrm{~g} / \mathrm{kg} /$ day in accordance with pharmacological experiments (9).

Fluoxetine capsules (2061AA; $20 \mathrm{mg}$ per capsule) were purchased from Eli Lilly Suzhou Pharmaceutical Group Co., Ltd., (Suzhou, China). The administration dose of fluoxetine was $1.8 \mathrm{~g} / \mathrm{kg} /$ day in accordance with previous pharmacological experiments (9).

Sucrose consumption test. The sucrose consumption test was performed as described previously (10). The test was conducted over 3 days. On day 1, rats were housed individually and had free access to two $100-\mathrm{ml}$ bottles of sucrose solution $(1 \% \mathrm{w} / \mathrm{v})$. Rats were trained to adapt to sucrose solution for $24 \mathrm{~h}$. On day 2, one bottle of sucrose solution was replaced with $100 \mathrm{ml}$ water for $24 \mathrm{~h}$. On day 3, rats were deprived of water and food for $23 \mathrm{~h}$, and subsequently received free access to two pre-weighed bottles containing $100 \mathrm{ml}$ sucrose solution (1\% w/v) and $100 \mathrm{ml}$ water, respectively. After $1 \mathrm{~h}$, the consumed volume from each bottle was recorded. The sucrose prefer- ence rate was calculated using the following formula: Sucrose preference rate $(\%)=$ Sucrose consumption/(water consumption + sucrose consumption) $\mathrm{x} 100$. This test was conducted prior to division of the rats into groups, and following the 28-day treatment period.

OFT. The OFT was performed according to a previously described method (11), to measure spontaneous activity in rodents. Briefly, the floor of a 100x100x40-cm square arena was divided into 16 equal $25 \times 25-\mathrm{cm}$ squares. A single rat was placed in the center of the arena, and after $30 \mathrm{sec}$ of adaptation the frequency of vertical and horizontal movement were recorded manually for $5 \mathrm{~min}$. All rat movement was recorded using an XZ-10 camera (Olympus Corporation, Tokyo, Japan) located $40 \mathrm{~cm}$ above the arena. After each test, the arena was cleaned with $90 \%$ alcohol solution.

Epilepsy model establishment. Adult male rats in the model, fluoxetine and CHSGS groups received $127 \mathrm{mg} / \mathrm{kg}$ lithium chloride (62480; Sigma-Aldrich, St. Louis, MO, USA) intraperitoneally (i.p.). On the following day, $1 \mathrm{mg} / \mathrm{kg}$ methylscopolamine bromide (W131102; i.p.; Shanghai Tongyong Pharmaceutical Co., Ltd., Shanghai, China) was administered to limit the peripheral effects of the convulsant. Status epilepticus was induced by injecting $35 \mathrm{mg} / \mathrm{kg}$ pilocarpine (P1650000; i.p.; Sigma-Aldrich) $30 \mathrm{~min}$ after the administration of methylscopolamine bromide. Animals were monitored throughout status epilepticus induction, and seizure severity was assessed according to the scale described by Racine (12). Animals that did not show clear signs of status epilepticus were excluded, since $\geq 1 \mathrm{~h}$ of status epilepticus is required to develop spontaneous recurrent epileptic seizures in the pilocarpine model of epilepsy. Status epilepticus occurred within 15-60 min and was characterized by continuous motor-limbic seizures accompanied by intermittent rearing and falling and occasional wild running spells. At $1 \mathrm{~h}$ after status epilepticus, the seizures were stopped with $4 \mathrm{mg} / \mathrm{kg}$ diazepam (AH131203; Shanghai Sunrise Pharmaceutical Co. Ltd., Shanghai, China) administered intramuscularly and/or $3 \mathrm{ml} / \mathrm{kg}$ chloral hydrate (i.p.; Anatomical Research Laboratory of Hunan University of Chinese Medicine).

Epilepsy with depression model establishment. Following the successful establishment of the epilepsy model, CMS was induced in accordance with a previously described method (13). The CMS regimen included seven different stressors, which were arranged in random order for 14 consecutive days as follows: Food and water deprivation ( $20 \mathrm{~h}$, followed by sucrose consumption test); water deprivation (18 h, followed by $1 \mathrm{~h}$ exposure to empty bottles); $45^{\circ}$ cage tilt $(17 \mathrm{~h})$; overnight illumination (lights on for a total of $36 \mathrm{~h}$ ); soiled cage (200 ml of water in $100 \mathrm{~g}$ sawdust bedding, $21 \mathrm{~h}$ ); swimming in $41^{\circ} \mathrm{C}$ water $(5 \mathrm{~min})$; and paired caging $(2 \mathrm{~h})$. At day 14 after the establishment of CMS, the model, fluoxetine and CHSGS groups were intragastrically perfused with $10 \mathrm{ml} / \mathrm{kg}$ normal saline, $1.8 \mathrm{~g} / \mathrm{kg}$ fluoxetine and $2.7 \mathrm{~g} / \mathrm{kg}$ CHSGS once per day for a total of 28 days.

5-Bromo-2'-deoxyuridine (BrdU) labeling and immunofluorescence staining. The thymidine analog BrdU (ED1100; 
Table I. Primer sequences used for polymerase chain reaction.

\begin{tabular}{llc}
\hline Primer & \multicolumn{1}{c}{ Sequences (5'-3') } & Product size (bp \\
\hline 5-HT1A & Forward: GCACCAGCTTAGGAACTTCG & 206 \\
& Reverse: CAGAGGAAGGTGCTCTTTGG & \\
$\beta$-actin & Forward: GTCAGGTCATCACTATCGGCAAT & 210 \\
& Reverse: AGAGGTCTTTACGGATGTCAACGT & \\
\hline
\end{tabular}

5-HT1A, 5-hydroxytryptamine 1A.

Sigma-Aldrich) was dissolved in saline with $0.007 \mathrm{M} \mathrm{NaOH}$. The BrdU solution $(50 \mathrm{mg} / \mathrm{kg}$ ) was administered twice per day for 7 days following the experimental treatment with fluoxetine or CHSGS administration. Subsequently, 6 rats in each group were sacrificed $24 \mathrm{~h}$ after the final BrdU administration using 10\% chloral hydrate, transfused intraperitoneally. Rats were perfused transcardially with cold $0.1 \mathrm{M}$ phosphate-buffered saline (PBS) and 4\% paraformaldehyde. The brains were removed, post-fixed for $18 \mathrm{~h}$, and placed in $30 \%$ sucrose until they sank. Frozen coronal sections were cut at $25 \mu \mathrm{m}$ using a cryostat (CM3050S; Leica Biosystems $\mathrm{GmbH}$, Nußloch, Germany) and stored at $-20^{\circ} \mathrm{C}$ in a cryoprotectant solution containing $25 \%$ ethylene glycol, $25 \%$ glycerol and $50 \% 0.1 \mathrm{M}$ phosphate buffer ( $\mathrm{pH}$ 7.4) for immunofluorescence staining.

The sections were subsequently incubated with $1 \%$ bovine serum albumin to block non-specific interactions. Following this step, the sections were incubated with mouse anti-rat BrdU monoclonal antibody (1:100; BM0201; Wuhan Boster Biological Technology, Ltd., Wuhan China) in PBS containing $0.3 \%$ Triton X-100 overnight at $4^{\circ} \mathrm{C}$. Subsequently, the sections were incubated with goat anti-mouse IgG-Cy2 (1:100; SA1021; Wuhan Boster Biological Technology, Ltd.) for $2 \mathrm{~h}$ at room temperature. The stained sections were observed using a BX-51 confocal microscope (Olympus Corporation). BrdU-labeled cells were quantified using Image Pro software, version 6.0 (Media Cybernetics, Inc., Bethesda, MD, USA).

RT-qPCR to detect 5-HTIA receptor mRNA expression levels in the rat hippocampus. Following the behavioral tests, 6 rats in each group were sacrificed. Fresh hippocampus tissue was dissected and placed on ice. Specimens were removed from the liquid nitrogen. Total RNA was extracted using TRIzol (2214L; Beijing CoWin Biotech Co., Ltd., Beijing, China). RNA concentration was determined using a P-Class NanoPhotometer (5622; Implen GmbH, München, Germany). The RT step was conducted using a Go ScriptTM Reverse Transcription System (0000112333; Promega Corporation, Madison, WI, USA), following the manufacturer's instructions. Primers were synthesized by Wuhan Boster Biological Technology, Ltd. (Table I), and a 2x SYBR GREEN-I Mix kit (0020140910; Beijing Bioteke Biotechnological Co., Ltd., Beijing, China) was used. Amplification reactions were conducted using a qPCR system (TL988; Xi'an Tianlong Science and Technology Co., Ltd., Xi'an, China).

The PCR cycling conditions were as follows: i) Pre-denaturation at $95^{\circ} \mathrm{C}$ for $10 \mathrm{~min}$; ii) 40 cycles of dena- turation at $95^{\circ} \mathrm{C}$ for $15 \mathrm{sec}$ and annealing/extension at $60^{\circ} \mathrm{C}$ for $1 \mathrm{~min}$; and iii) final extension at $60^{\circ} \mathrm{C}$ for $5 \mathrm{~min}$. The specificity of PCR products was monitored via melting curve analysis, and the standard curves of the target and reference gene $\beta$-actin were prepared. The ratio of 5-HT1A to $\beta$-actin was considered as the relative expression of the target gene. Relative quantification of gene expression was determined using the $2^{-\Delta \Delta \mathrm{Cq}}$ method.

Statistical analysis. Statistical analysis was performed using SPSS software, version 17.0 (SPSS, Inc., Chicago, IL, USA). Measurement data are expressed as the mean \pm standard error of the mean. Analysis of variance was used for intergroup comparisons. $\mathrm{P}<0.05$ was considered to indicate a statistically significant difference.

\section{Results}

Animal grouping and treatments. A total of $60 \mathrm{SD}$ rats were used. The baseline scores of the sucrose consumption test and OFT were determined, and then 48 SD rats with similar behavior were identified and randomly divided into four groups: Control, model, fluoxetine and CHSGS groups ( $\mathrm{n}=12$ per group).

Behavior of epileptic rats with depression. After 28 days of treatment, the consumption of sucrose water, the sucrose preference rate, and frequency of vertical and horizontal movement decreased in the model group compared with the control group $(\mathrm{P}<0.05)$. Compared with the control group, frequency of vertical movement decreased in the fluoxetine group $(\mathrm{P}<0.05)$, while frequency of vertical and horizontal movement decreased in the CHSGS group $(\mathrm{P}<0.05)$. Compared with the model group, all sucrose and OFT test parameters were increased in the fluoxetine and CHSGS groups $(\mathrm{P}<0.05)$. However, no statistically significant differences were observed between the fluoxetine and CHSGS groups (Fig. 1).

CHSGS promotes cellular proliferation in the hippocampal dentate gyrus. Immunofluorescence staining indicated the presence of BrdU-labeled cells in the hippocampal dentate gyrus (Fig. 2). After 28 days of treatment, the number of BrdU-labeled cells increased significantly in the hippocampal dentate gyrus of the model, fluoxetine and CHSGS groups compared with the control group $(\mathrm{P}<0.05)$. Furthermore, compared with the model group, the number of BrdU-labeled 

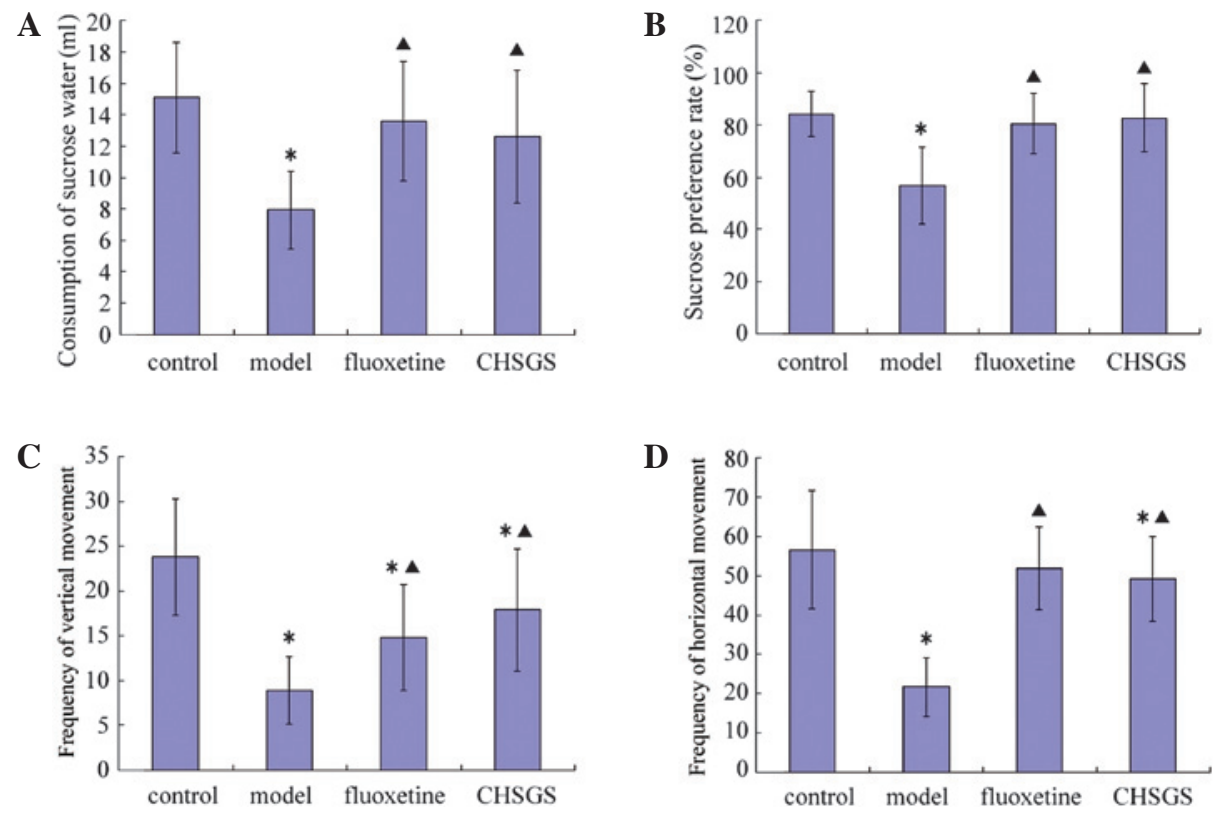

Figure 1. Comparison of behavioral difference in the (A and B) sucrose consumption test and (C and D ) open-field test (frequency/5 min). Data expressed as the mean \pm standard error $(n=12)$. ${ }^{*} \mathrm{P}<0.05$ vs. control group. ${ }^{\mathbf{\Delta}} \mathrm{P}<0.05$ vs. model group. CHSGS, Chaihu-Shugan-San.

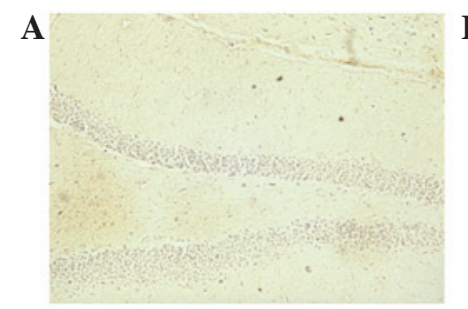

$\mathbf{E}$

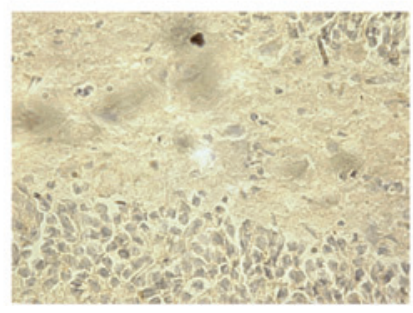

$\mathbf{B}$
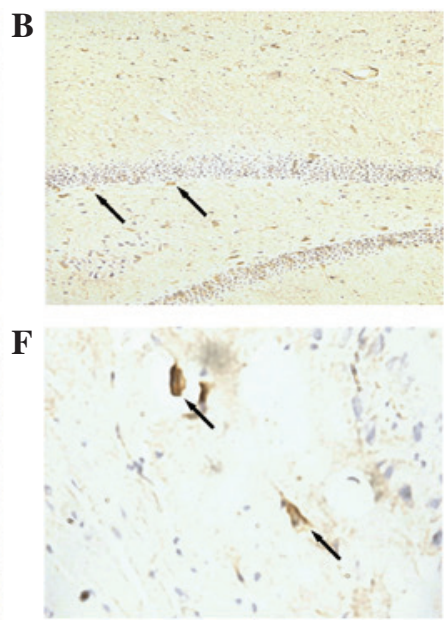
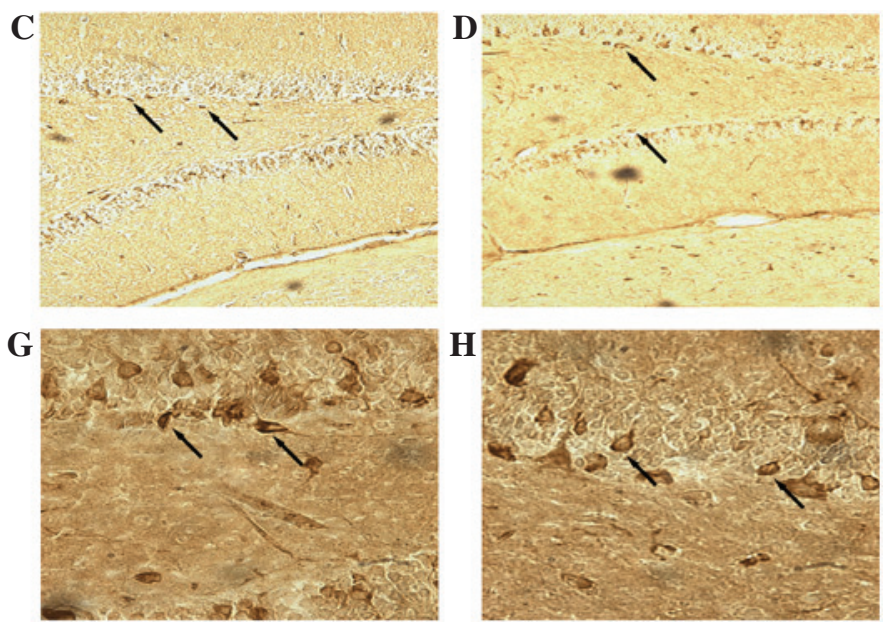

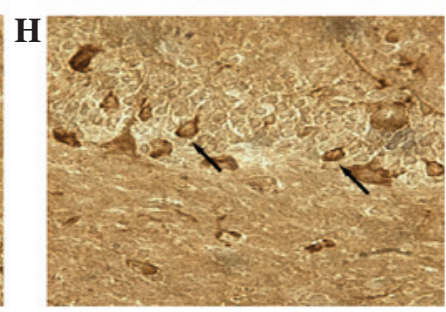

Figure 2. Representative 5-bromo-2'-deoxyuridine (BrdU) immunofluorescence images of the dentate gyrus in different groups following 28 days of treatment obtained by confocal microscopy. The (A and E) control group exhibits fewer BrdU-labeled cells compared with the (B and F) model, (C and G) fluoxetine and (D and H) Chaihu-Shugan-San groups. Black arrows indicate BrdU-labeled cells in the hippocampal dentate gyrus. Images presented at (A-D) x100 and (E-H) x400 magnification.

cells increased significantly in the hippocampal dentate gyrus in the fluoxetine and CHSGS groups $(\mathrm{P}<0.05)$. No significant difference was identified between the fluoxetine and CHSGS groups ( $\mathrm{P}>0.05$; Fig. 3 ).

CHSGS enhances the $m R N A$ expression of 5-HTIA receptor in the hippocampal dentate gyrus. Following 28 days of treatment, the expression levels of 5-HT1A receptor mRNA decreased in the model, fluoxetine and CHSGS groups compared with the control group $(\mathrm{P}<0.05)$. Compared with the model group, the expression levels of 5-HT1A receptor mRNA were enhanced in the fluoxetine and CHSGS groups $(\mathrm{P}<0.05)$. However, no difference in 5-HT1A expression levels was observed between the fluoxetine and CHSGS groups $(\mathrm{P}>0.05$; Figs. 4 and 5).

\section{Discussion}

The reported prevalence of epilepsy in China ranges between 3.6 and $7.0 \%$ (14). The number of patients with active epilepsy was $>9$ million in 2010, and increases by $\sim 400,000$ each year. Recurrent epileptic attacks and long-term treatment can seriously reduce the life quality of the patients, and affect mentality to a certain extent. Evident psychological and behavioral disorders have been identified in large numbers of patients with epilepsy (15). The prevalence of depression in patients with epilepsy is reportedly $23.1 \%$, which is higher compared with that in the general population. In addition, the lifetime prevalence of depression in patients with epilepsy is high, approaching 13\% (16). Thus, the co-occurrence of epilepsy and depression is a common phenomenon. 


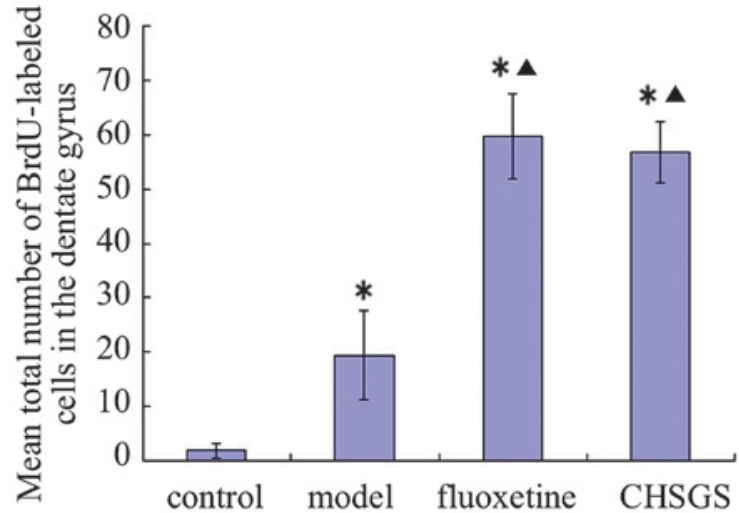

Figure 3. Mean total number of BrdU-labeled cells in the dentate gyrus of different groups following 28 days of treatment. Data expressed as the mean \pm standard error $(\mathrm{n}=6)$. ${ }^{*} \mathrm{P}<0.05$ vs. control group. ${ }^{\Delta} \mathrm{P}<0.05$ vs. model group. BrdU, 5-bromo-2'-deoxyuridine; CHSGS, Chaihu-Shugan-San.

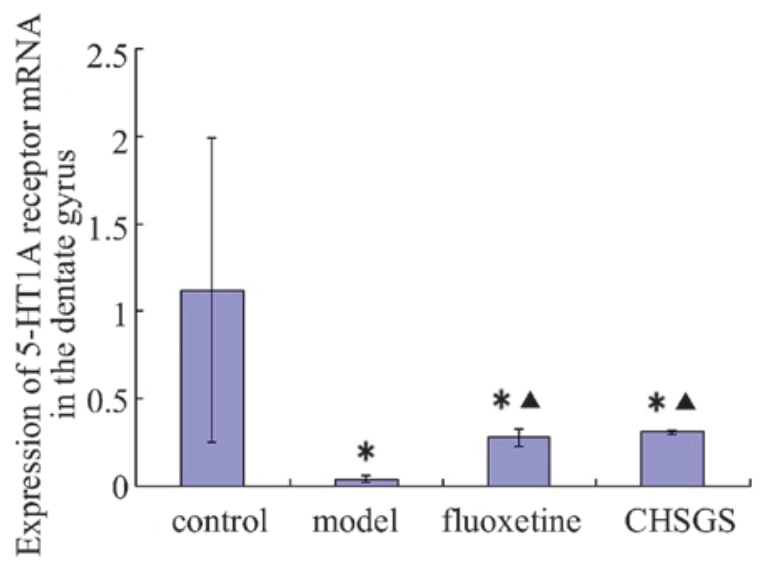

Figure 4. Comparison of the expression of 5-HT1A receptor mRNA in the dentate gyrus following 28 days of treatment. Data expressed as the mean \pm standard error $(\mathrm{n}=6)$. ${ }^{*} \mathrm{P}<0.05$ vs. control group. ${ }^{\Delta} \mathrm{P}<0.05$ vs. model group. 5-HT1A, 5-hydroxytryptamine 1A; CHSGS, Chaihu-Shugan-San.

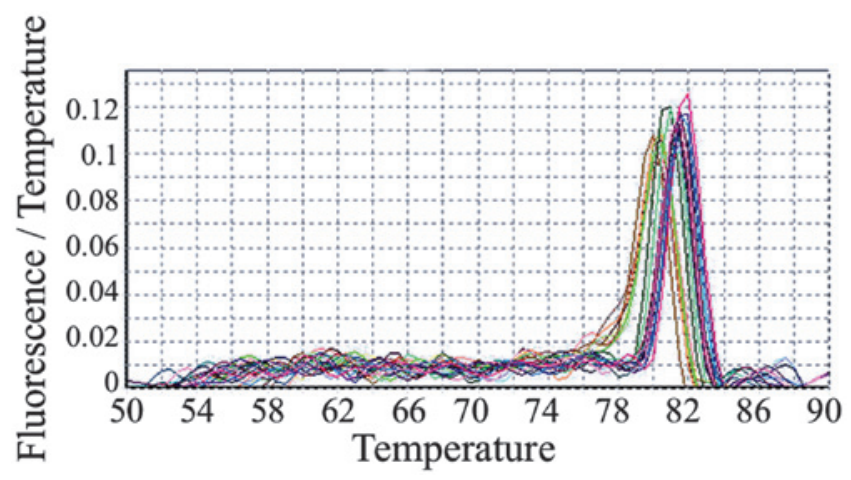

Figure 5. Polymerase chain reaction (PCR) amplification curve of 5-hydroxytryptamine $1 \mathrm{~A}$ receptor mRNA and $\beta$-actin gene. Melting curve shows a single peak, indicating single PCR products.

In our previous study, epileptic rats with depression were used in a lithium pilocarpine-induced epileptic model (4). Thus, a combined model of epilepsy with depression was established. However, the model exhibited a number of shortcomings, such as the low success rate of model establishment of $\sim 25 \%$, high experimental expense and waste of resources. In the present study, the establishment of the combined model of epilepsy with depression has been improved compared with the previous model. The revised model includes a combination of the traditional lithium pilocarpine-induced epilepsy model and the CMS-induced depression model. The success rate of model establishment reached $\sim 80 \%$ in the present study.

In our previous study, the 5-HT1A receptor agonist 8-OH-DPAT was used to improve depressive symptoms in epileptic rats with depression. However, the clinical application of 8-OH-DPAT is currently limited, which may be due to a number of factors. Firstly, although 5-HT1A receptor agonists, such as tandospirone, have good antidepressant effects (17), they are frequently used as antidepressant synergists (18). Secondly, the treatment compliance for traditional and newer antidepressant drugs has been adversely affected by their defects, including side effects and addictive properties. Certain antidepressant agents in clinical use, such as tricyclic antidepressants, bupropion and fluoxetine $(19,20)$, have been reported to cause seizures in certain patients. Therefore, the selection of antidepressants for epileptic patients with depression is controversial.

According to Traditional Chinese Medicine (TCM) theory, epilepsy with depression belongs to the 'depressed syndrome' category of diseases (21) and the pathogenesis has a marked association with emotion. The liver fails to maintain the normal flow of 'Qi', which affects 'pneuma' transportation to the five 'zang' organs and the six 'fu' organs, resulting in corresponding emotional symptoms and potentially depression. The primary treatment method for depression according to the TCM tradition is the herbal preparation Shu Gan, which is hypothesized to conduct 'Qi' and release 'liver depression'. According to TCM theory, the movement of 'Qi' is able to 'free the blood', and thus alleviate various forms of stagnancy. Therefore, in the present study a representative decoction of the 'Shu Gan Li Qi' method (CHSGS) was selected as a candidate treatment for epileptic rats with depression.

It has been reported that CHSGS reduces the expression of microRNA-125a and microRNA-182, which are associated with the onset of depression (22) in the hippocampus. Furthermore, CHSGS increases brain-derived neurotrophic factor and tropomyosin receptor kinase B levels in the amygdaloid nucleus, hippocampus and frontal lobe (23) and upregulates the expression of ERK1/2 mRNA in the hippocampus (24), thus improving the symptoms of depression in rat depression models. CHSGS appears to enhance the expression levels of B-cell lymphoma 2 (Bcl-2) and decrease those of Bcl-2-like protein 4 in the hippocampus and frontal cortex, thus inhibiting neuronal apoptosis in epileptic rats (25). In addition, CHSGS has been shown to decrease the expression of multidrug resistance protein P-glycoprotein and electroencephalograph epileptiform discharges in the hippocampus and temporal lobe cortex (26), which are associated with antiepileptic changes. In the clinical treatment of patients with post-stroke depression, the curative effect of CHSGS was found to be better compared with fluoxetine at relieving the symptoms of depression and stabilizing patient emotional state (27). CHSGS combined with the conventional antidepressant venlafaxine has been shown to significantly improve neurological functions and alleviate depressive symptoms in 
stroke patients (28). In addition, Wang et al (29) conducted a meta-analysis which confirmed the efficacy and safety of CHSGS for the treatment of depression. In the present study, CHSGS was administered to epileptic rats with depression. No statistically significant difference in depressive behavior was observed between the CHSGS and fluoxetine groups. By contrast, compared with the model group, the behavioral data in CHSGS group exhibited statistically significant differences. These results suggest that CHSGS improves the symptoms of depression in epileptic rats with depression.

However, the underlying mechanism by which CHSGS ameliorates the depressive symptoms in epileptic rats with depression requires clarification. Recent studies and our previous study have indicated that the 5-HT1A receptor is associated with the pathogenesis of epilepsy with depression $(4,30)$. The 5-HT1A receptor has become a key drug target for the treatment of epilepsy with depression (31). Therefore, it is possible that CHSGS improves depressive symptoms in rats by modifying the expression of the 5-HT1A receptor. In the present study, compared with the control group, the expression of 5-HT1A receptor mRNA was decreased in the model, fluoxetine and CHSGS groups. The expression of 5-HT1A receptor mRNA was enhanced in the fluoxetine and CHSGS groups compared with the model group. However, no significant difference in 5-HT1A receptor mRNA expression was identified between the fluoxetine and CHSGS groups. These results indicate that the expression of 5-HT1A receptor mRNA is reduced in epileptic rats with depression. Fluoxetine and CHSGS are able to increase the expression of 5-HT1A receptor mRNA; however, there is no discernible difference in their therapeutic efficacy.

The mechanism underlying the antiepileptic and antidepressive effect of increased 5-HT1A receptor expression remains unclear. Our previous study indicated that the 5-HT1A receptor is associated with neural plasticity and is crucially involved in the pathogenesis of epilepsy with depression (4). Therefore, we hypothesize that the 5-HT1A receptor exerts its antiepileptic and antidepressive effect by promoting neural plasticity. The adult hippocampus has the capability to generate new neurons throughout life, and this property contributes to functional plasticity under physiological and pathological conditions (32). Further research has shown that neural stem cells are present in the subgranular zone of the dentate gyrus throughout the lifetime of an individual (33). These cells proliferate and differentiate into neurons and glial cells, and subsequently migrate into the granule cell layer and finally into the molecular layer (33). Various antidepressants, including tricyclic antidepressants and selective serotonin reuptake inhibitors, are able to increase neuronal proliferation in the hippocampal dentate gyrus in adult rodents (34). A previous study has indicated that the activation of the 5-HT1A receptor is a critical step in the activation of seizure-induced cellular proliferation and survival in the dentate gyrus (35). This effect may attenuate depressive symptoms in epilepsy by enhancing neuronal plasticity.

In the present study, no statistically significant difference was detected between the CHSGS and fluoxetine groups with regard to the number of BrdU-labeled cells, indicating that the two agents have similar effects on cell proliferation. Cell proliferation in the CHSGS group was significantly greater than that in the model group, and the expression of 5-HT1A receptor mRNA was increased accordingly. Thus, the results of the present study indicate that CHSGS regulates neurogenesis in epileptic rats with depression by adjusting the expression levels of the 5-HT1A receptor. However, the exact mechanism by which CHSGS exerts its anti-epileptic and anti-depressive effect against epilepsy with depression remains unclear and requires further study.

\section{Acknowledgements}

This study was supported by grants from the National Natural Science Foundation of China (No. 81302899 and 81373551), Key Science and Research Program of Hunan Department of Science and Technology (No. 2012TF-1005), Foundation of Shanxi Province for Returnees (No. 2008-52) and the Project of Hunan Provincial Education Department (No. 15C1051).

\section{References}

1. Peng WF and Wang X: Comorbidity of epilepsy and depression: From clinical to basic research. Shi Jie Lin Chuang Yao Wu 33: 13-17, 2012 (In Chinese).

2. Pineda EA, Hensler JG, Sankar R, Shin D, Burke TF and Mazarati AM: Plasticity of presynaptic and postsynaptic serotonin 1A receptors in an animal model of epilepsy-associated depression. Neuropsychopharmacology 36: 1305-1316, 2011.

3. Theodore WH, Wiggs EA, Martinez AR, Dustin IH, Khan OI, Appel S, Reeves-Tyer P and Sato S: Serotonin 1A receptors, depression, and memory in temporal lobe epilepsy. Epilepsia 53: 129-133, 2012.

4. Yang P, Sun MZ, Li L and Shen YH: 8-hydroxy-dipropylaminotetralin promotes neural plasticity in epileptic rats with depression. Neural Regen Res 7: 565-571, 2012.

5. Al-Abri SA, Orengo JP, Hayashi S, Thoren KL, Benowitz NL and Olson KR: Delayed bupropion cardiotoxicity associated with elevated serum concentrations of bupropion but not hydroxybupropion. Clin Toxicol (Phila) 51: 1230-1234, 2013.

6. Wang S, Hu SY, Zhang CH, Qiu J and Li YH: Antidepressant-like activity of Chaihu-Shugan-San aqueous extract in rats and its possible mechanism. Pharmacogn Mag 10 (Suppl 1): S50-S56, 2014.

7. The Ministry of Science and Technology of the People's Republic of China: Guidance Suggestions for the Care and Use of Laboratory Animals. Beijing, 2006.

8. Chinese Pharmacopoeia Commission: Pharmacopoeia of the People's Republic of China. Vol 1. 10th edition. Chinese Medical Science and Technology Press, Beijing, pp38-263, 2010.

9. Tan YZ: Pharmacology Experiment. People's Medical Publishing House, Beijing, pp141-142, 2008.

10. Wang SH, Zhang ZJ, Guo YJ, Teng AJ and Chen BA: Expression of 5-hydroxytryptamine 1A receptor protein and message RNA in the dentate gyrus in post-stroke depression rats. Zhong Hua Jing Shen Ke Za Zhi 41: 107-110, 2008 (In Chinese).

11. Meng H, Wang Y, Huang M, Lin W, Wang S and Zhang B: Chronic deep brain stimulation of the lateral habenula nucleus in a rat model of depression. Brain Res 1422: 32-38, 2011.

12. Racine RJ: Modification of seizure activity by electrical stimulation. II. Motor seizure. Electroencephalogr Clin Neurophysiol 32: 281-294, 1972.

13. Wang SH, Zhang ZJ, Guo YJ, Sui YX and Sun Y: Notch1 signaling related hippocampal neurogenesis in adult poststroke depression rats: A valid index for an efficient combined citalopram and WAY100635 pharmacotherapy. Behav Pharmacol 21: 47-57, 2010.

14. Zhou Y, Liu M and Liang WN: Progress on the epidemiological study of epilepsy. Zhonghua Liu Xing Bing Xue Za Zhi 28: 92-94, 2007 (In Chinese).

15. Lacey CJ, Salzberg MR and D'Souza WJ: Risk factors for depression in community-treated epilepsy: Systematic review. Epilepsy Behav 43: 1-7, 2015. 
16. Fiest KM, Dykeman J, Patten SB, Wiebe S, Kaplan GG, Maxwell CJ, Bulloch AG and Jette N: Depression in epilepsy: A systematic review and meta-analysis. Neurology 80: 590-599, 2013.

17. Zhao YL and Yang X: Comparative observation on synergistic effect of tandospirone antidepressant. Lin Chuang Jing Shen Yi Xue Za Zhi 18: 40, 2008 (In Chinese).

18. Mago R, Mahajan R and Thase ME: Medically serious adverse effects of newer antidepressants. Curr Psychiatry Rep 10: 249-257, 2008

19. Ma L and Wu HF: A case of epileptic state caused by fluoxetine. Lin Chuang Wu Zhen Wu Zhi 21: 80, 2008 (In Chinese).

20. Pineda EA, Hensler JG, Sankar R, Shin D, Burke TF and Mazarati AM: Interleukin-1 $\beta$ causes fluoxetine resistance in an animal model of epilepsy-associated depression. Neurotherapeutics 9: 477-485, 2012.

21. Jiang GQ, Wang JM, Fan MY, Lv JJ and Zhang HK: Progress on pathogenesis and treatment of epilepsy with depression in integrative medicine. Zhong Xi Yi Jie He Xin Nao Xue Guan Bing Za Zhi 9: 1247-1249, 2011 (In Chinese).

22. Cao MQ, Chen DH, Zhang CH and Wu ZZ: Screening of specific microRNA in hippocampus of depression model rats and intervention effect of Chaihu Shugan San. Zhongguo Zhong Yao Za Zhi 38: 1585-1589, 2013 (In Chinese).

23. Deng Y, Zhang CH and Zhang HN: Effects of chaihu shugan powder on the behavior and expressions of BDNF and TrkB in the hippocampus, amygdala and the frontal lobe in rat model of depression. Zhongguo Zhong Xi Yi Jie He Za Zhi 31: 1373-1378, 2011 (In Chinese).

24. Wang S, Hu S, Zhang C, Qiu J and Li Y: Effect of Chaihu Shugan San and its components on expression of ERK1/2 mRNA in the hippocampus of rats with chronic mild unpredicted stress depression. Zhong Nan Da Xue Xue Bao Yi Xue Ban 36: 93-100, 2011 (In Chinese).

25. Huang YS, Zhuo Y and Liu ZL: Effect of Chaihu-Shugan-San on expression of bc1-2 and bax in cardiazole-induced epileptic rats hippocampus and frontal cortex. Zhonghua Lin Chuang Yi Shi Za Zhi (Dian Zi Ban). 6: 1574-1575, 2012 (In Chinese).
26. Xie W, Shi GJ, Li CZ, Bao Y, Yu L, Yu YH and Du NN: Effect of Chaihu Shugan Tang on electroencephalogram and expression of multidrug resistance protein p-glycoprotein of refractory epilepsy. Zhong Guo Shi Yan Fang Ji Xue Za Zhi 17: 128-131, 2011 (In Chinese).

27. Liu J and Zhong C: 40 cases of post-stroke depression treated by Chaihu-Shugan-San. Zhong Guo Zhong Yi Ji Zheng 21: 788, 2012 (In Chinese)

28. Zou LH, Li H, Zeng ZQ, Chen XD and Deng ZH: Chaihu Shugan powder combined with venlafaxine for treatment of post-stroke depression. Zhong Xi Yi Jie He Xin Nao Xue Guan Bing Za Zhi 9: 1330-1332, 2011 (In Chinese).

29. Wang Y, Fan R and Huang X: Meta-analysis of the clinical effectiveness of traditional Chinese medicine formula Chaihu-Shugan-San in depression. J Ethnopharmacol 141: 571-577, 2012.

30. Kanner AM: Depression and epilepsy: A bidirectional relation? Epilepsia 52 (Suppl 1): S21-S27, 2011.

31. Hasler G, Bonwetsch R, Giovacchini G, Toczek MT, Bagic A, Luckenbaugh DA, Drevets WC and Theodore WH: 5-HT1A receptor binding in temporal lobe epilepsy patients with and without major depression. Biol Psychiatry 62: 1258-1264, 2007.

32. Covic M, Karaca E and Lie DC: Epigenetic regulation of neurogenesis in the adult hippocampus. Heredity (Edinb) 105: 122-134, 2010.

33. Ming GL and Song H: Adult neurogenesis in the mammalian brain: Significant answers and significant questions. Neuron 70: 687-702, 2011.

34. Taciany Bonassoli V, Micheli Chassot J, Longhini R, Milani H, Mello JC and de Oliveira RM: Subchronic administration of Trichilia catigua ethyl-acetate fraction promotes antidepressant-like effects and increases hippocampal cell proliferation in mice. J Ethnopharmacol 143: 179-184, 2012.

35. Radley JJ and Jacobs BL: Pilocarpine-induced status epilepticus increases cell proliferation in the dentate gyrus of adult rats via a 5-HT1A receptor-dependent mechanism. Brain Res 966: 1-12, 2003. 